\title{
3D Multi-scale Modeling Of Early Stage Chick Limb Development
}

Jeyaraman Srividhya, John Scott Gens and James A. Glazier

The Biocomplexity Institute, Indiana University, Bloomington, IN 47405 USA

Limb development has been one of the focus of research in the US since the Thalidomide disaster of the 1950s and 60s, in which children of $\mathrm{m}$ others who took the drug for $\mathrm{m}$ orning sickness, were born with truncated limbs. This was a $\mathrm{m}$ ajor spur to modern developmental toxicity regulation. First step in addressing the toxicity is to th oroughly understand the re gulatory processes invo lved in the limb development. Experimental evidence from both the mouse and chick model system $\mathrm{s}$ has contributed greatly to our understanding of the developm ental processes involved. In em bryonic chicks, the wing buds begin to form as a sligh $t$ swelling in the lateral body wall, adjacent to the $15^{\text {th }}$ through the $20^{\text {th }}$ somites, at Ha mburger-Hamilton $(\mathrm{HH})$ stage 16 [1]. The growth res ults from an increa se in the proliferation of limb bud mesenchyme relative to the su rrounding flank mesenchyme[2] (Figure 1A). Recent evidence suggests th at fibroblast growth factors (FGFs) secreted by a specialized ectodermal tissue called "Apical Ectodermal Ridge" (AER), play a $\mathrm{m}$ ajor role in prom oting cell proliferation of mesenchyme [3]. AER is a pseudo-stratified epithelial tissue that is formed at the interface of the fringe expressing dorsal ectoderm and fringe non-expressing ventral ectoderm via Notch-Delta ligand formation[4]. From HH stages 23-24, the limbs grow into asymmetrical "paddles".

In this paper we present a 3D multi-scale model of the early stage (HH16 to HH23) limb development showing the limb outgrowth us ing a m ulti-cell modeling platform Compucell3D(C C3D) (www.compucell3d.org)[5]. CC3D allows us to virtually represent cells using lattice points in space, for which cell-like properties can be defined. Our mode 1 is built upon th ree major components integrated together to give rise to overall limb outgrowth: (a) First, at st age HH16, the AER is form ed through inter- and intra-cellular Notch-Delta signaling - modeled using ordinary differential equations (ODEs ), (b) Growth and proliferation of the $\lim \mathrm{b}$ mesenchyme promoted by the FGF8, sec reted by the newly formed AER - m odeled using CC 3D, and (f) Grow th of ectoderm over limb mesenchyme - modeled with a specifically developed contact inhibition mechanism using CC3D.

(a) AER formation through Notch-Delta Signaling: Based on the literature, we have developed a mechanism (Figure 1B) and an ODE $\mathrm{m}$ odel through wh ich inter-cellular Notch-Delta signaling gives rises to high Notch activity only at the boundary, transforming the normal ectodermal cells in to Apical Ectodermal cells. The ODE model is integrated with CC3D platform using BionetSolver [6].

(b) Growth and proliferation of limb mesenchyme: The form ed AER secretes the morphogen FGF8. The FGF8 diffuses through the limb mesenchyme promoting proliferation. We simulate the diffusion of FGF8 using partial dif ferential equations (PDEs). The mesenchyme is set to grow and multiply at a constant rate in response to an FGF8 gradient threshold.

(c)Growth of Ectoderm over mesenchyme: As the limb outgrows, the ectoderm also expands along with the growing m esenchyme. The ectoderm grows in an "as needed" basis when stretched by the underlying limb mesenchyme. We have developed a specific approach where the ectodermal cells grow based on the loss cell-cell contact area. The rate of growth of the ectoderm al cell volume is set as in equation (1), which allows the ectoderm to grow over the m esenchyme without any tears, thereby covering the limb outgrowth. 
$\frac{\mathrm{d} V_{\text {cell }}}{\mathrm{d} t}=k_{m} \times\left(\frac{\alpha_{c}{ }^{n}\left(1-\alpha^{n}\right)}{\alpha_{c}{ }^{n}+\alpha^{n}}\right) \times\left(\frac{\beta^{m}}{\beta_{c}^{m}+\beta^{m}}\right)\left(\frac{\beta_{c}^{m}+0.5^{m}}{0.5^{m}}\right)$

$\alpha=\frac{\text { Ectoderm-Ectoderm contact area }}{\text { Ectoderm cell surface area }}, \beta=\frac{\text { Ectoderm-Mesenchyme contact area }}{\text { Ectoderm cell surface area }}$

\section{Results}

By successfully integrating methods (a) through (c), we have computationally simulated the early

A

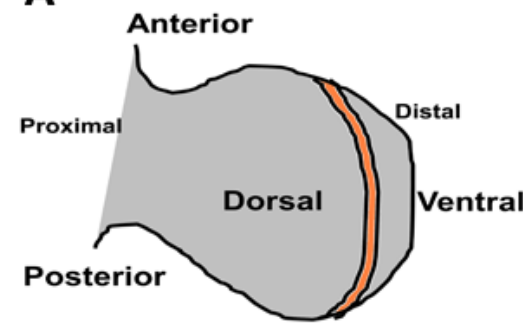

C

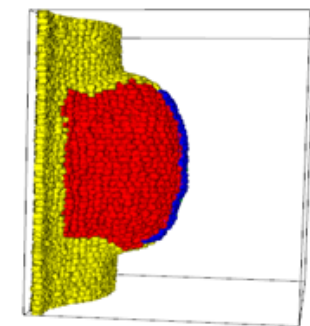

B Dorsal Cells

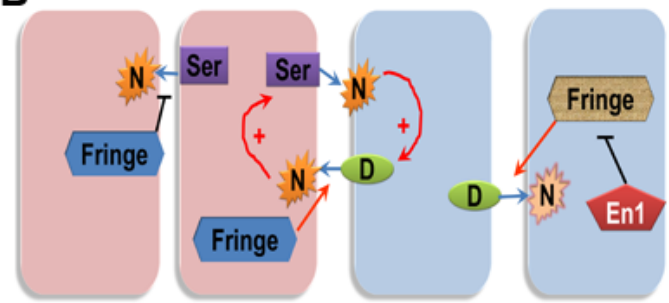

D

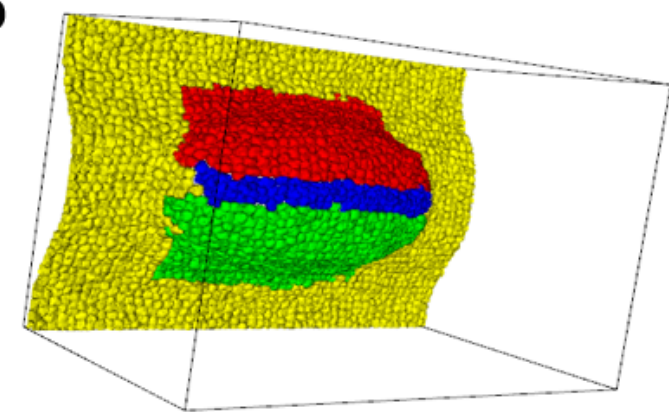

Figure 1 A. Illustration of limb. B. Mechanism for AER formation involving Fringe, $\operatorname{Notch}(\mathrm{N})$, Serrate (Ser) and Delta(D), C. Simulation showing proximo distal outgrowth of the limb. D. Alternate view of the simulation of limb bud showing AER (blue), dorsal ectoderm (red) and ventral ectoderm (green), and limb ectoderm (yellow).

development of chicklimb in CC3D platform (Figure 1C and D). We show the limb bud outgrowth along with the AER in two different view $\mathrm{s}$. We have used $m$ esenchymal cell diffusion data as well as real cell cycle tim es to scale our simulations to the expe riments in the lite rature. At stage $\mathrm{HH} 23$, the length of the $\lim \mathrm{b}$ bud in the proxim odistal (PD) direction is equal to the length in the Ante rior-Posterior (AP) direction. However, our simulations produce a lim $b$ outgrowth whose PD length is smaller than observed limb PD length, showing that there are mechanisms other than sim ple proliferation are involved. W e then include cell-intercalation in the mesenchyme in addition to proliferation and show that the PD length of the limb increases compared to growth obtained using proliferation alone.

Our model is the first multi-scale computational effort that depicts the complex fundamental processes involved in the early limb developm ent including the inter- and intr a-cellular signaling for the AER formation. Our model can be extended to contain complex signaling mechanisms and has the potential to be developed into a complex predictive model for toxicological studies.

\section{References}

[1] V Hamburger and H L Hamilton, Developmental Dynamics. 195 (1992) p. 231-272.

[2] R L Searls and M Y Janners, Developmental Biology. 24 (1971) p. 198-213.

[3] P H Crossley, et al., Cell. 84 (1996) p. 127-136.

[4] E Laufer, et al., Nature. 386 (1997) p. 366-373.

[5] M H Swat, et al., Multi-Scale Modeling of Tissues Using CompuCell3D, in Methods In Cell Biology, A.R. Asthagiri and A.P. Arkin, Editors. 2012, Academic Press, USA. p. 325-366.

[6] H M Sauro, et al., OMICS. 7 (2003) p. 355-72.

[7] The authors acknowledge the EPA grant - The Texas-Indiana Virtual STAR Center; Data-Generating in vitro and in silico Models of Developmental Toxicity in Embryonic Stem Cells and Zebrafish. 